\title{
LAS LUCHAS POR LA JUSTICIA AMBIENTAL en la Argentina sojera
}

\section{Amalia Leguizamón ${ }^{*}$}

Resumen: Este trabajo explora la distribución desigual de los beneficios, así como los costos sociales y ambientales del modelo sojero en Argentina y su impacto en el surgimiento de las luchas sociales por la justicia ambiental. En la década de 1990 Argentina adoptó un modelo agroindustrial neoliberal basado en la producción de soja transgénica para la exportación. Aunque celebrado como un éxito, la expansión de los monocultivos de soja transgénica ha acarreado graves impactos socioecológicos. Diversos actores sociales han comenzado a movilizarse en contra de la injusticia ambiental resultante. El artículo se centra en el movimiento campesino-indígena del norte de Argentina que pelea por sus derechos territoriales y en los movimientos en contra de las fumigaciones con agroquímicos en la región central pampeana. Dichos grupos, relativamente sin poder para controlar los recursos donde viven, reciben poco o ningún beneficio de la producción de soja transgénica en su entorno $y$, sin embargo, soportan la mayor parte de los costos sociales y ecológicos. Tales luchas vinculan el bienestar ambiental y social, convirtiéndose en pugnas por la sustentabilidad ecológica, la justicia social y la equidad.

Palabras clave: soja, transgénicos, Argentina, movimientos socioambientales, justicia ambiental.

* Profesora asistente del Departamento de Sociología y el Centro Stone de Estudios Latinoamericanos, Tulane University, Estados Unidos. Correo-e: aleguiza@tulane.edu 


\title{
ENVIRONMENTAL INJUSTICE IN ARGENTINA:
}

struggles against genetically modified soy

\begin{abstract}
This paper explores the unequal distribution of the environmental and social costs and benefits of the genetically modified (GM) soy model in Argentina and its impact on grievance formation and the emergence of contestation. In the 1990s, Argentina transitioned into a neoliberal agro-industrial model based on producing GM soy for export. Though celebrated as a success, the expansion of GM soy monocultures has brought widespread socio-ecological disruption. Various social actors have started to mobilize against the resulting environmental injustice. I focus on the peasant-indigenous movement in the north of the country, which is struggling for land rights, and the movements against agrochemical spraying in the central Pampas region. These groups, which are relatively powerless to control resources where they live, and that experience little or no benefit from GM soy production, nevertheless bear most of its social and ecological costs. These struggles link environmental and social well-being, becoming struggles for ecological sustainability as well as social justice and equity.
\end{abstract}

Keywords: soybeans, GM, Argentina, mobilization, environmental justice. 


\section{Introducción}

A comienzos del siglo XXI aumentó el número de conflictos socioambientales en América Latina y en el Sur global, a medida que más comunidades se han organizado para resistir los proyectos extractivistas promovidos por gobiernos y corporaciones bajo la lógica neoliberal (Martínez, 2014). El extractivismo es un modelo de desarrollo socioeconómico basado en la explotación intensiva de recursos naturales, facilitada por avances técnico-científicos, y en la consecuente exportación de estos commodities, materias primas con poco o ningún valor agregado. Si bien el extractivismo no es nuevo para la región, la reestructuración neoliberal de las últimas tres décadas significó un retorno a ese modelo, impulsado por gobiernos de izquierda y derecha y, a menudo, administrado por corporaciones transnacionales (Gudynas, 2014; Otero y Lapegna, 2016).

Una de las formas que adquiere el extractivismo en Argentina es la producción agroindustrial a gran escala de soja transgénica para la exportación (Cáceres, 2015), la cual fue adoptada en 1996 como una estrategia de acumulación clave de la reestructuración neoliberal de esa década, que continuaba bajo los gobiernos posneoliberales de Néstor y Cristina Kirchner (Torrado, 2016). Argentina es el tercer productor $y$ exportador mundial de soja luego de Estados Unidos y Brasil. Las exportaciones de soja y sus derivados (grano, harina, aceite y biodiésel) representan cerca de una cuarta parte del comercio exterior de ese país. El cien por ciento de la soja sembrada es transgénica; con cosechas y ganancias récord año tras año, el modelo sojero es ampliamente celebrado como un éxito (Leguizamón, 2014a).

Sin embargo, el auge de la soja también ha traído consigo graves problemas sociales y ambientales. Desde la introducción de la biotecnología transgénica en 1997, un promedio de 726 mil ha se añadió a la producción anual de soja. En 2015, 20 millones de hectáreas (52 por ciento de la tierra 
cultivada en Argentina) fueron sembradas con soja transgénica (Ministerio de Agricultura, Ganadería y Pesca, 2015). El dramático crecimiento de la producción ha expandido la frontera agrícola desde la Pampa - zona central y principal región agroexportadora- hacia el norte, principalmente sobre el monte chaqueño; ello propicia un aumento en la tasa de deforestación y violentos desplazamientos de familias campesinas e indígenas, así como un incremento en el riesgo ambiental y de la salud pública debido a las fumigaciones con agroquímicos.

La presente investigación se enfoca en los movimientos suscitados en Argentina para resistir la injusticia ambiental, esto es, para protestar en contra de los problemas sociales y ambientales del modelo sojero y transgénico. La justicia ambiental (environmental justice) destaca las asimetrías de poder en el acceso y la distribución de los «bienes»y «males» ambientales con respecto a desigualdades estructurales de raza, etnia, clase y género (Mohai, Pellow y Roberts, 2009). En Estados Unidos y América Latina múltiples movimientos por la justicia ambiental han surgido a medida que comunidades relativamente sin poder para controlar los recursos de los lugares donde viven - y que sufren de manera directa los riesgos ambientales de la producción y el uso de esos recursos-, enmarcan las consecuencias negativas como un problema y se organizan para remediarlas (Carruthers, 2008).

No se trata de una cuestión de blanco o negro, los matices son importantes. En primer lugar, no todas las comunidades que sufren el riesgo ambiental protestan (Auyero y Swistun, 2009; Lapegna, 2015). En segundo lugar, ni la distribución de costos y beneficios ni la distribución del poder en la sociedad es un juego de suma cero. Es extraño que en Argentina el modelo sojero frecuentemente se presente como un caso de «ganadores»y «perdedores» (véase como ejemplo González, 2009). Esta descripción enfatiza la gran división surgida de la reorganización de la agricultura argentina: por un lado, una elite formada por el Estado y las 
corporaciones del agronegocio que se beneficia con la producción de soja (Newell, 2009); por el otro, los pequeños agricultores, los trabajadores rurales y los pueblos campesinos e indígenas que, como consecuencia, terminan en peores condiciones (Lapegna, 2014).

Al mismo tiempo existe un amplio sector en el medio, en particular medianos productores y propietarios, que se beneficia directa e indirectamente de la producción de soja al arrendar la tierra (Gras, 2009). No obstante, quienes estudian dichos temas destacan que la producción agrícola en Argentina se concentra en forma creciente en manos de grandes empresas del agro transnacional, lo cual acelera los procesos de acumulación por desposesión (Cáceres, 2015; Gras y Hernández, 2014; Leguizamón, 2016). Así, aunque el espectro del poder es amplio y variado, a medida que el modelo se intensifica las zonas grises se oscurecen, hecho que vuelve al fenómeno más relevante y urgente.

En Argentina, las luchas contra el extractivismo son notorias con relación a la minería. La resistencia contra el modelo sojero, aunque menos generalizada, también está creciendo. El objetivo de este estudio es documentar esas pugnas contra el despojo. Con base en datos recogidos entre 2011 y 2015 a través de entrevistas, ${ }^{1}$ investigación de archivos y datos secundarios, se explora la distribución desigual de los costos y beneficios ambientales y sociales del modelo de la soja transgénica en Argentina, su impacto en la formación de reclamos y la emergencia de la protesta social.

Se destacan dos aspectos: 1) el movimiento campesino-indígena en el norte de Argentina, que se opone a la biotecnología transgénica como

\footnotetext{
${ }^{1}$ Las entrevistas tuvieron lugar entre 2011-2012 y 2015 con miembros del Mocase-VC, Paren de Fumigar, Córdoba; Paren de Fumigarnos, Santa Fe; Asamblea UAC Buenos Aires; Basta de Fumigarnos, Las Palmas-La Leonesa; Madres de Barrio Ituzaingó; Asamblea Malvinas Lucha por la Vida; y con los investigadores Norma Giarracca y Miguel Teubal (Universidad de Buenos Aires), Cecilia Canevari (Universidad de Santiago del Estero), Mauricio Berger y Cecilia Carrizo (Universidad de Córdoba).
} 
un aspecto de la lucha contra la globalización neoliberal («antineoliberalismo»); 2) las «asambleas ciudadanas por la salud y la vida», surgidas en su mayoría en la región central de la Pampa, en respuesta al aumento de enfermedades relacionadas con las fumigaciones («antiagrotóxicos»). Las comunidades tienen escaso poder para controlar los recursos donde viven y cosechan poco o ningún beneficio de la producción local de soja transgénica, sin embargo, soportan la mayor parte de sus costos socioecológicos. El cuadro 1 resume las características fundamentales de ambos movimientos socioambientales.

\section{Cuadro 1}

Movimientos sociales antisoja transgénica en Argentina

\begin{tabular}{|c|c|c|}
\hline & Antineoliberalismo & Antiagrotóxicos \\
\hline $\begin{array}{l}\text { Actores } \\
\text { principales }\end{array}$ & $\begin{array}{l}\text { Campesinos } \\
\text { y poblaciones indígenas }\end{array}$ & $\begin{array}{l}\text { Ciudadanos, Vecinos: } \\
\text { zonas periurbanas } \\
\text { y habitantes rurales } \\
\text { que no viven de la } \\
\text { producción rural }\end{array}$ \\
\hline $\begin{array}{l}\text { Sitio de luchal } \\
\text { Ecorregión }\end{array}$ & Chaco (norte) & Pampas (central) \\
\hline $\begin{array}{l}\text { Principal } \\
\text { agravio }\end{array}$ & $\begin{array}{l}\text { Desalojos forzados y } \\
\text { violentos; antitransgénicos } \\
\text { como parte de una lucha } \\
\text { más amplia contra } \\
\text { el neoliberalismo }\end{array}$ & $\begin{array}{l}\text { Efectos de la fumigación } \\
\text { agroquímica en la salud } \\
\text { (agrotóxicos) }\end{array}$ \\
\hline $\begin{array}{l}\text { Objetivo } \\
\text { central }\end{array}$ & $\begin{array}{l}\text { Obtener control sobre } \\
\text { la tierra y el territorio }\end{array}$ & $\begin{array}{l}\text { Paren de Fumigar: } \\
\text { regular el uso } \\
\text { de agroquímicos }\end{array}$ \\
\hline Alcance & $\begin{array}{l}\text { Local, con conexiones } \\
\text { transnacionales } \\
\text { (La Vía Campesina) }\end{array}$ & $\begin{array}{l}\text { Local, con algunas } \\
\text { alianzas regionales } \\
\text { y nacionales }\end{array}$ \\
\hline
\end{tabular}


Los contextos de resistencia en los que surgen estos movimientos son muy diferentes. Referente a la agricultura argentina, una distinción básica debe hacerse entre la región pampeana y el resto del país. Las llanuras centrales se han dedicado históricamente a la producción agraria capitalista orientada al mercado de exportación; el ecosistema fue arrasado para la producción agroindustrial desde tiempos de la Colonia. Cabe mencionar que 87 por ciento de la producción de soja se lleva a cabo en esa región, en las provincias de Buenos Aires, Entre Ríos, Santa Fe, Córdoba y La Pampa (Ministerio de Agricultura, Ganadería y Pesca, 2015). En el resto del país la agricultura se orienta a la producción de alimentos para el consumo interno. La región del Chaco, en el norte, es un bosque seco que atraviesa un proceso de rápido desmonte para dar espacio a la producción de soja, y que está habitado en su mayoría por pueblos campesinos e indígenas. A diferencia de la Pampa, el suelo en el Chaco no es apto para la agricultura industrial a gran escala.

Este texto es una contribución a los estudios críticos sobre el surgimiento de movimientos antitransgénicos en el Sur global (Scoones, 2008; Gutiérrez \& Fitting, 2016); se suma a otros esfuerzos para establecer un diálogo entre debates y teorías acerca del ecologismo en América Latina y Estados Unidos (Carruthers, 2008; Martínez, 2014). Es indispensable vincular tales enfoques para replantear las cuestiones del poder, la sustentabilidad ecológica y las dinámicas del cambio tecnológico y agrario.

\section{Pueblos campesinos e indígenas versus el neoliberalismo}

La mayoría de las familias campesinas e indígenas en Argentina vive en el norte, en las provincias de Salta, Jujuy, Tucumán, Chaco, Santiago del Estero, Formosa y Misiones. Muchas de esas familias se han movilizado para reclamar derechos sobre la tierra, una demanda que se ha 
acrecentado con la expansión de la soja transgénica en su territorio (Domínguez, 2009). En palabras de los miembros del Movimiento Campesino de Santiago del Estero:

Nosotros vivimos este avance de la frontera del agronegocio, la soja, los cultivos transgénicos (...) La amenaza permanente en el tema de los territorios, de las tierras de las comunidades campesinas-indígenas, la contaminación ambiental por los productos del paquete tecnológico que traen los cultivos transgénicos, la amenaza a la biodiversidad, la contaminación ambiental, la pérdida de nuestras semillas, la criminalización de nuestras luchas por defender el territorio (Entrevista con Mocase-VC, agosto 2011).

Las protestas de las comunidades campesinas e indígenas contra la soja transgénica se inscriben dentro de una demanda más amplia por el control del territorio y sus riquezas naturales. La lucha por la tierra surge en paralelo con la identidad campesino-indígena y su organización como movimiento social (Teubal, 2009).

El Movimiento Campesino de Santiago del Estero (Mocase) es el mayor y más importante movimiento campesino en Argentina; comenzó a finales de 1970 como respuesta a los desalojos forzados por la agroindustria (Lapegna, 2013). En la década de 1990, en lo más profundo de la reestructuración neoliberal, el movimiento se organizó como Mocase. Para 2003 se dividió en Mocase-PSA, una facción cercana al gobierno, y en Mocase-VC, aliado con el movimiento transnacional La Vía Campesina (Barbetta, 2005). El núcleo de la agenda del Mocase-VC es la lucha por el territorio, una demanda protegida por la «ley veinteañal», que otorga derechos de propiedad a quienes han vivido y trabajado un terreno por más de veinte años. Este reclamo se une al de los derechos de propiedad por los derechos ancestrales como pueblos nativos. 
En territorio campesino-indígena, la expansión de los monocultivos de soja transgénica se traduce en desmontes, tala y quema del bosque para hacer espacio a la agricultura industrial a gran escala. Desde la década de 1970 la frontera agraria se expandió de la región pampeana hacia el monte, el bosque seco del norte chaqueño. El Chaco es uno de los ecosistemas forestales más grandes de América Latina y una de las fronteras de deforestación más activas en el mundo. Con la expansión de la soja como impulso principal, entre 1972 y 2011, 2.7 millones de hectáreas del monte chaqueño han sido deforestadas; 64 por ciento han tenido lugar a partir de la introducción de la biotecnología transgénica en 1997 (Gasparri, Grau y Gutiérrez, 2013, apéndice).

La deforestación ocasiona pérdidas ecológicas y pone en peligro la biodiversidad (Pengue, 2005). Es también una notable fuente de emisiones de carbono, lo que contribuye al cambio climático (Gasparri, Grau y Manghi, 2008) y acarrea pérdidas sociales. Para las comunidades campesinas e indígenas el monte es un bien común, pues les proporciona leña para cocinar y vender (en forma de carbón) y sirve de espacio de pastoreo para los animales domésticos. El monte es históricamente una fuente de empleo para los hombres que se desempeñan como hacheros; los pueblos indígenas lo consideran una fuente de sustento y supervivencia, así como de su cultura y su identidad. «Nos arrebatan la tierra y la identidad», me decían los miembros del Mocase. Las demandas sociales y ambientales se unen, el reclamo de proteger el medio ambiente se entrelaza con el de reivindicar la identidad, la dignidad y la soberanía.

Durante la entrevista, los miembros del Mocase-VC relataron los desalojos violentos que acompañan a las deforestaciones; las topadoras que pasan sobre el monte y los hogares, y las fuerzas paramilitares que amenazan, e incluso asesinan, a sus líderes (Lapegna, 2013). Las familias campesinas e indígenas carecen de poder para enfrentar la agroindustria: en los 224 conflictos acerca de la tenencia de la tierra relevados en 2013 
—2.8 millones de hectáreas en la región del Chaco-casi 18 mil familias campesinas e indígenas se vieron afectadas, de las cuales la mitad posee 50 ha o menos (Red Agroforestal Chaco Argentina, 2013).

Para el Mocase-VC el objetivo principal y más urgente es «que las familias se queden en su tierra». Con el propósito de defender el territorio campesino, el movimiento participa de varias formas de acción colectiva, desde la acción directa para resistir los desalojos forzados hasta las acciones legales a fin de otorgar derechos territoriales a las familias. Asimismo, el Mocase-VC pone en marcha un modelo agrario alternativo que busca el desarrollo local; implica el uso de métodos agroecológicos y la creación de una red de comercio justo para la producción campesina. Estos proyectos son alternativas que transforman la dinámica sociedad-ecosistema más allá de la lógica del extractivismo.

En síntesis, para el Mocase-VC y otros movimientos campesino-indígenas de América Latina, la lucha contra la soja transgénica surge de la necesidad de proteger la propia vida y el sustento, además de resistir los procesos de acumulación por desposesión derivado de la implementación de programas neoliberales en la región. La biotecnología transgénica es sólo un aspecto de un modelo económico basado en el extractivismo. Por lo anterior, el Mocase-VC denuncia la agroindustria en su totalidad, junto con otras actividades extractivas como la minería. La lucha contra los transgénicos se inscribe dentro de una pugna más general que reclama el control local, y no extranjero, de los recursos naturales. ${ }^{2}$

${ }^{2}$ Esta es la perspectiva que los líderes de dichos movimientos presentan en el discurso público. Otros trabajos muestran que pueblos campesinos e indígenas del Sur global tienen una relación compleja y con otros matices respecto a la biotecnología transgénica en su vida cotidiana; por ejemplo, en Argentina, véase Lapegna (2014), en México, Fitting (2011), y en India, Roy (2015). 


\section{Asambleas ciudadanas versus agrotóxicos}

El agroquímico más utilizado en Argentina y alrededor del mundo es el glifosato, un herbicida de amplio espectro desarrollado y comercializado por Monsanto bajo la marca Roundup (Cressey, 2015; GRR, 2006). Es parte de un «paquete tecnológico» que se usa a la par de la maquinaria de siembra directa y las semillas de soja Roundup Ready, también de Monsanto (diseñadas para tolerar la pulverización con glifosato).

Un argumento relevante esgrimido al momento de adoptar la biotecnología de semillas transgénicas en Argentina era que su empleo iba a reducir la aplicación de agroquímicos más tóxicos que el glifosato, incluso en un inicio, la adopción de la soja transgénica disminuyó el uso de agroquímicos (Trigo, 2011); sin embargo, al aumentar el área plantada con soja transgénica y al aparecer supermalezas resistentes al glifosato, el volumen total de agroquímicos utilizados se elevó (Catacora et al., 2012). Supermalezas resistentes al glifosato obligan a los productores a incrementar el uso de herbicidas, a fumigar con más frecuencia y a combinar glifosato con herbicidas aún más tóxicos: 2,4-D, dicamba, paraquat, atrazina y endosulfán (Binimelis, Pengue y Monterroso, 2009). Como resultado, el empleo de glifosato por agricultores argentinos pasó de 821 mil kilogramos en 1996 —cuando sólo 6 por ciento de la superficie plantada de soja era transgénica- a 88 millones en 2014, aplicado sobre una superficie de 20 millones de hectáreas de soja, cien por ciento transgénica (Benbrook, 2016, anexo 1).

La espectacular expansión del uso de glifosato acrecienta el riesgo de impacto ecológico y sobre la salud pública (Benbrook, 2016). Estudios de laboratorio muestran que las formulaciones de glifosato y Roundup tienen efectos perturbadores del sistema endocrino en ratas y embriones de rata, lo que provoca deficiencias crónicas en riñones, hígado y graves malformaciones (Paganelli et al., 2010; Séralini et al., 2014). Médicos argentinos han documentado un incremento en las tasas de abortos espontáneos 
y malformaciones en recién nacidos entre mujeres con antecedentes de exposición directa a fumigaciones en todo el país (Red Universitaria de Ambiente y Salud, 2010). La Organización Mundial de la Salud reclasificó en 2015 al glifosato como «probablemente cancerígeno» (Cressey, 2015).

En consecuencia, en Argentina y en el mundo, expertos y legos por igual se están organizando para resistir los peligros ambientales y de salud causados por las fumigaciones con agroquímicos (Arancibia, 2013; Ezquerro, 2016). Un conjunto de madres argentinas que vive en el barrio Ituzaingó Anexo, en el conurbano de la capital de Córdoba, fue uno de los primeros en tomar acuerdos en contra de las fumigaciones. Desde entonces varios grupos en todo el país se han unido a la demanda de «Paren de Fumigar». En Malvinas Argentinas, a 20 kilómetros del barrio Ituzaingó Anexo, vecinos afectados por la fumigación de cultivos en campos aledaños intentan detener la construcción de una planta de Monsanto en su ciudad. Tales luchas se describen a continuación.

\section{Madres de Barrio Ituzaingó Anexo}

En nuestro caso, se han violado los derechos humanos y lo que nosotros estamos pidiendo es un derecho a la salud, a la vida y a un medio ambiente sano, que son prácticamente, si vos te das cuenta, un derecho que tendríamos que tener todos y ahora no lo tenemos. Es algo intransferible el derecho a la vida, y violar ese derecho, que es un derecho humano, hace mal a todo; han pisoteado lo más sagrado de una persona. Ellos, con las fumigaciones, nos han fumigado con avionetas, no les importó la gente, nadie fue capaz de decir: no, hay un barrio cerca (...) No, si no salía la gente, si no salíamos nosotros a pelear, esto todavía lo seguirían haciendo. Se frenaron gracias a la pelea. La organización surgió porque tuvimos que defendernos de algo que nos estaba dañando (madre del barrio Ituzaingó, en Carrizo y Berger, 2009: 13). 
Las Madres de Barrio Ituzaingó Anexo fueron las primeras en oponerse a las fumigaciones con glifosato en Argentina. Se trata de un barrio de clase trabajadora en el cordón industrial de la ciudad de Córdoba, rodeado de campos de soja. La movilización comenzó en 2002, cuando un grupo de madres se percató de que muchos de sus niños y vecinos del barrio enfermaban y morían. En una población de 5 mil habitantes se revelaron más de doscientos casos de cáncer, enfermedades respiratorias y de la piel, abortos espontáneos y malformaciones en niños y bebés.

Dichas madres han trabajado extensa y duramente para demostrar la relación entre las fumigaciones con agroquímicos y el envenenamiento de su comunidad (Carrizo y Berger, 2009). Su trabajo comenzó de manera directa con los vecinos a fin de denunciar explícitamente la fumigación de agrotóxicos como un riesgo a la salud. Desde entonces, como parte de su repertorio de protesta, las madres marchan en la plaza San Martín de la Ciudad de Córdoba con sus niños y familiares enfermos, sus bocas cubiertas por barbijos, en una actuación que recuerda las marchas de las madres de Plaza de Mayo. Una demanda clave es el cumplimiento de las ordenanzas locales que prohíben las fumigaciones a menos de 2 mil 500 metros de áreas pobladas. Como una circunstancia histórica, las madres exhibieron una demanda judicial en 2012 contra un productor de soja y un fumigador por contaminación dolosa con agroquímicos.

En colaboración con expertos, las madres han desarrollado una «epidemiología popular» para demostrar los vínculos entre las enfermedades y la fumigación con agroquímicos (Arancibia, 2013). Las pruebas de laboratorio muestran la existencia de endosulfán en el suministro de agua y residuos de productos agroquímicos en los cuerpos de los residentes del barrio. Relevamientos reflejan una prevalencia inusualmente alta de patologías en el barrio: anemia, linfoma, leucemia y cáncer, agrupadas en pocas cuadras (Carrizo y Berger, 2009). El mapeo de patrones de enfermedad es un método epidemiológico y toxicológico importante utilizado 
también por muchos movimientos de justicia ambiental en Estados Unidos (McCormick, 2015).

\section{Paren de Fumigar}

En 2006, en solidaridad con las Madres de Barrio Ituzaingó, un grupo de académicos y activistas con sede en Buenos Aires llamado Grupo de Reflexión Rural (GRR) organizó una campaña contra la fumigación de agroquímicos. Esta campaña, denominada Paren de Fumigar, se resume en el informe Pueblos fumigados (GRR, 2006), que reúne las experiencias y demandas de residentes afectados en la región pampeana.

El objetivo de la campaña del GRR era relevar los pueblos fumigados para organizarlos en un solo grupo y así coordinar esfuerzos y recursos. Esta iniciativa presentó la magnitud del problema, una condición esencial para enmarcar el tema como un conflicto social en otras ciudades. En una reunión clave en Colonia Caroya, Córdoba, en 2008, la campaña coordinada por GRR se transformó en un colectivo de diferentes capítulos. Si bien existen intercambios entre los grupos y las asambleas (por correo electrónico y reuniones), todavía no existe una red nacional; hay alianzas con diversos grupos que se organizan en torno a las luchas socioambientales bajo el nombre de Unión de Asambleas Ciudadanas (UAC).

En la mayoría de los pueblos rurales argentinos no han surgido protestas en contra de los agroquímicos, incluso en lugares donde las fumigaciones son percibidas como potencialmente peligrosas (Lapegna, 2015; Leguizamón, 2014b). Sin embargo, son cada vez más los grupos de Pueblos Fumigados que surgen en las provincias de Córdoba, Santa Fe, Entre Ríos y Buenos Aires, ahí proliferan los campos de soja. Protestas en contra de las fumigaciones también han tenido lugar en la provincia de Chaco, en particular en las localidades de La Leonesa y Las Palmas. 
A diferencia de los que componen el movimiento campesino-indígena, los miembros de Paren de Fumigar no son trabajadores ni productores rurales, y aunque muchos de ellos viven en zonas rurales rodeados de campos agrícolas, por lo general no se benefician directamente con la agricultura. Durante el trabajo de campo encontré a maestros, estudiantes, abogados, médicos y amas de casa que se identificaron como vecinos autoconvocados; una declaración destinada a separarse de partidos y afiliaciones políticas y con la defensa de la salud y la vida de sus hijos como única agenda. Esta forma de organización alrededor del riesgo a la salud, en especial a los niños, es característica de luchas simbólicas por la justicia ambiental en Estados Unidos, por ejemplo en Love Canal en el estado de Nueva York (Levine, 1982).

\section{Malvinas Argentinas le dice «No a Monsanto»}

En junio de 2012, año en que las madres de Ituzaingó comenzaron su juicio histórico, la presidenta Cristina Fernández de Kirchner anunció una nueva inversión de Monsanto en Argentina: la construcción de una planta de semillas de maíz transgénico, la segunda más grande de América Latina. La planta se ubicaría en la localidad rural de Malvinas Argentinas, a 20 kilómetros del barrio Ituzaingó.

Los habitantes de Malvinas Argentinas, la ciudad más pobre de la provincia de Córdoba, que ya sufrían los efectos de la fumigación en campos aledaños, decidieron organizarse y decirle «No a Monsanto» y a la instalación de una planta tóxica en su ciudad. Lo que inició como una coalición de vecinos a mediados de 2012, en apenas un año se convirtió en un bloqueo y ocupación que sigue en pie en junio de 2016 y que ha frenado efectivamente los planes de construcción de Monsanto. 


\section{Conclusiones}

La expansión de la soja transgénica en Argentina se celebra como un éxito, aunque este boom haya traído también graves consecuencias sociales y ecológicas. Las topadoras arrasan el monte del norte chaqueño para hacer espacio a más soja, lo que devasta ecosistemas y desplaza violentamente a las poblaciones locales. Aviones fumigadores esparcen tóxicos agroquímicos sobre los pueblos que bordean los campos de soja y arriesgan la salud de quienes allí viven. El presente trabajo destaca cómo los costos y los beneficios del modelo de la soja transgénica se han distribuido de manera desigual: los que tienen menor poder relativo para decidir acerca del modelo extractivista y beneficiarse con él son los que sobrellevan la mayor parte de sus costos.

En ese sentido, la investigación se centra en los movimientos socioambientales que han surgido para protestar por la injusticia ambiental resultante del modelo de la soja transgénica en Argentina. En concreto, se puntualiza la manera en que los pueblos campesinos e indígenas en el norte del país se movilizan para defender sus derechos territoriales contra la usurpación llevada a cabo por la agroindustria. Asimismo, se hace hincapié en el movimiento antiagrotóxico, así como en las asambleas de vecinos autoconvocados contra las fumigaciones de agroquímicos, los cuales pelean por el derecho a vivir en un medio ambiente saludable. Aunque estos dos movimientos se constituyen por diferentes actores y sus demandas difieren, proteger el derecho a la vida y la subsistencia es su causa común. Al fusionar las peticiones ambientales con las pugnas sociales se conjuga un movimiento por la justicia ambiental del Sur global.

La pugna es muy desigual: campesinos que se enfrentan a topadoras, madres y estudiantes que se oponen a Monsanto. Esto no hace que su combate sea menos urgente; al contrario, los modelos de desarrollo basados en el extractivismo sólo expanden y aceleran la degradación socioecológica. 
El marco teórico de la justicia ambiental resalta la forma en que las poblaciones locales, que sufren el impacto directamente en sus cuerpos y vidas, devienen los más conspicuos actores en las organizaciones de ese tipo. Con relación a la sustentabilidad, las comunidades locales son clave para mantener el diálogo sociedad-naturaleza (Gould, 2006). Puesto que la promesa de movimientos de justicia ambiental es fusionar el bienestar ambiental con el social, las luchas por la justicia social y la equidad también se convierten en pugnas por la sustentabilidad ecológica.

\section{Referencias}

Arancibia, F. (2013). «Challenging the Bioeconomy: The Dynamics of Collective Action in Argentina». Technology in Society 35(2).

Auyero, J. \& Swistun, D.A. (2009). Flammable: Environmental Suffering in an Argentine Shantytown. Oxford: Oxford University Press.

Barbetta, P. (2005). «El movimiento campesino de Santiago del Estero: luchas y sentidos en torno a la problemática de la tierra». En Giarracca, N. \& Teubal, M. (eds.). El campo argentino en la encrucijada. Buenos Aires: Alianza.

Benbrook, C.M. (2016). «Trends in Glyphosate Herbicide Use in the United States and Globally». Environmental Sciences Europe 28(3).

Binimelis, R., Pengue, W. \& Monterroso, I. (2009). «Transgenic Treadmill: Responses to the Emergence and Spread of Glyphosate-Resistant Johnsongrass in Argentina». Geoforum 40(4).

Cáceres, D.M. (2015). «Accumulation by Dispossession and Socio-Environmental Conflicts Caused by the Expansion of Agribusiness in Argentina». Journal of Agrarian Change 15(1).

Carrizo, C. \& Berger, M. (2009). Estado incivil y ciudadanos sin Estado: paradojas del ejercicio de derechos en cuestiones ambientales. Córdoba: Narvaja Editor. 
Carrizo, C. \& Berger, M. (2012). «Citizens' Rights and Environmental Genocide». Environmental Justice 5(2).

Carruthers, D.V. (2008). Environmental Justice in Latin America: Problems, Promise, and Practice. Cambridge: MIT Press.

Catacora Vargas, G. et al. (2012). Soybean Production in the Southern Cone of the Americas: Update on Land and Pesticide Use. Bolivia: Virmegraf.

Cressey, D. (March 24, 2015). «Widely Used Herbicide Linked to Cancer». Nature News.

Domínguez, D.I. (2009). La lucha por la tierra en Argentina en los albores del siglo XXI. La recreación del campesinado y de los pueblos originarios. Argentina: Universidad de Buenos Aires.

Ezquerro Cañete, A. (2016). «Poisoned, Dispossessed and Excluded: A Critique of the Neoliberal Soy Regime in Paraguay». Journal of Agrarian Change, http://onlinelibrary.wiley.com/doi/10.1111/joac.12164/abstract

Fitting, E. (2011). The Struggle for Maize: Campesinos, Workers, and Transgenic Corn in the Mexican Countryside. Durham: Duke University Press.

Gasparri, N.I., Grau, H.R. \& Gutiérrez Angonese, J. (2013). «Linkages between Soybean and Neotropical Deforestation: Coupling and Transient Decoupling Dynamics in a Multi-Decadal Analysis». Global Environmental Change 23(6).

Gasparri, N.I., Grau, H.R. \& Manghi, E. (2008). «Carbon Pools and Emissions from Deforestation in Extra-Tropical Forests of Northern Argentina between 1900 and 2005». Ecosystems 11(8).

González Arzac, R. (2009). ¡Adentro! millonarios, chacareros y perdedores en la nueva Argentina rural. Buenos Aires: Marea.

Gould, K.A. (2006). «Promoting Sustainability». En Blau, J.R. \& Iyall Smith, K.E. (eds.), Public Sociologies Reader. Lanham: Rowman \& Littlefield.

Gras, C. (2009). «Changing Patterns in Family Farming: The Case of the Pampa Region, Argentina». Journal of Agrarian Change 9(3). 
Gras, C. \& Hernández, V.A. (2014). «Agribusiness and Large-Scale Farming: Capitalist Globalisation in Argentine Agriculture». Canadian Journal of Development Studies 35(3).

Grupo de Reflexión Rural (2006). «Pueblos fumigados: informe sobre la problemática del uso de plaguicidas en las principales provincias sojeras de la Argentina», http://www.grupodereflexionrural.com/trabajos/ Plaguicidas\%20en\%20la\%20Argentina.pdf

Gudynas E. (2014). «Conflictos y extractivismos: conceptos, contenidos y dinámicas». DECURSOS, Revista en Ciencias Sociales, 27-28.

Gutiérrez Escobar, L.M. \& Fitting, E. (2016). «The Red de Semillas Libres: Contesting Biohegemony in Colombia». Journal of Agrarian Change, http://onlinelibrary.wiley.com/doi/10.1111/joac.12161/abstract

Lapegna, P. (2013). «The Expansion of Transgenic Soybeans and the Killing of Indigenous Peasants in Argentina». Societies without Borders 8(2).

Lapegna, P. (2014). «Transgénicos, agroquímicos y campesinas/os en Argentina: escalas globales y locales, dinámicas de resistencia y adaptación». En Otero, G. (ed.), La dieta neoliberal. México: Universidad Autónoma Metropolitana.

Lapegna, P. (2015). «Genetically Modified Soybeans, Agrochemical Exposure and Everyday Forms of Peasant Collaboration in Argentina». Journal of Peasant Studies, http://www.tandfonline.com/doi/abs/10.1080/03066150.2 015.1041519

Leguizamón, A. (2014). «Modifying Argentina: GM Soy and Socio-Environmental Change». Geoforum 53.

Leguizamón, A. (2014b). Roundup Ready Nation: The Political Ecology of Genetically Modified Soy in Argentina. New York: University of New York.

Leguizamón, A. (2016). «Disappearing Nature? Agribusiness, Biotechnology, and Distance in Argentine Soybean Production». Journal of Peasant Studies 43(2). 
Levine, A. (1982). Love Canal: Science, Politics and People. Lanham: Lexington Books.

Martínez Alier, J. (2014), «The Environmentalism of the Poor». Geoforum 54.

McCormick, S. (2015). «The Sociology of Environmental Health». En Gould, K.A. \& Lewis, T.L. (eds.). Twenty Lessons in Environmental Sociology. New York: Oxford University Press.

Ministerio de Agricultura, Ganadería y Pesca (2015). «Sistema integrado de información agropecuaria», http://www.siia.gov.ar/series

Mohai, P., Pellow, D.N. \& Roberts, J.T. (2009). «Environmental Justice». Annual Review of Environment and Resources 34.

Newell, P. (2009). «Bio-Hegemony: The Political Economy of Agricultural Biotechnology in Argentina». Journal of Latin American Studies 41.

Otero, G. \& Lapegna, P. (2016). «Transgenic Crops in Latin America: Expropriation, Negative Value and the State». Journal of Agrarian Change, http://onlinelibrary.wiley.com/doi/10.1111/joac.12159/full

Paganelli, A., Gnazzo, V., Acosta, H., López, S.L. \& Carrasco, A.E. (2010). «Glyphosate-Based Herbicides Produce Teratogenic Effects on Vertebrates by Impairing Retinoic Acid Signaling». Chemical Research in Toxicology 23(10).

Pengue, W.A. (2005). «Transgenic Crops in Argentina: The Ecological and Social Debt». Bulletin of Science, Technology E Society 25(4).

Red Agroforestal Chaco Argentina (2013). Conflictos sobre la tenencia de tierra y ambientales en la región del Chaco Argentino: tercer informe. Santa Fe: REDAF.

Red Universitaria de Ambiente y Salud (2010). «Primer Encuentro Nacional de Médicos de Pueblos Fumigados», http://www.reduas.com.ar/infor me-encuentro-medicos-pueblos-fumigados/

Roy, D. (2015). «Contesting Corporate Transgenic Crops in a Semi-Peripheral Context: The Case of the Anti-GM Movement in India». Journal of World-Systems Research 21(1). 
Scoones, I. (2008). «Mobilizing against GM Crops in India, South Africa and Brazil». Journal of Agrarian Change 8(2).

Séralini, G.E. et al. (2014). "Republished Study: Long-Term Toxicity of a Roundup Herbicide and a Roundup-Tolerant Genetically Modified Maize». Environmental Sciences Europe 26(1).

Teubal, M. (2009). «Agrarian Reform and Social Movements in the Age of Globalization». Latin American Perspectives 36(4).

Torrado, M. (2016). «Food Regime Analysis in a Post-Neoliberal Era: Argentina and the Expansion of Transgenic Soybeans». Journal of Agrarian Change, http://onlinelibrary.wiley.com/doi/10.1111/joac.12158/full

Trigo, E.J. (2011). Fifteen Years of Genetically Modified Crops in Argentine Agriculture, www.argenbio.org/adc/uploads/15_years_Executive_summary_of_ GM_crops_in_Argentina.pdf 